\title{
L'influence de la culture dans l'expression verbale de la douleur : étude comparative entre des patients cancéreux français et syriens.
}

\author{
LEBREUILLY Romain \\ Maitre de conférences en Psychologie \\ Laboratoire InterPsy (EA 4432), groupe GRC, Nancy \\ SAKKOUR Sam \\ Psychologue
}

Centre d'Etude et de Recherche sur les Risques et les Vulnérabilités (CERReV), EA 3919, Université de Caen Basse- Normandie, France.

\section{LEBREUILLY Joëlle}

Maître de conférences

Centre d'Etude et de Recherche sur les Risques et les Vulnérabilités (CERReV), EA 3919, Université de Caen Basse- Normandie, France. 


\section{Résumé}

Cette étude examine le rôle de la culture dans l'expression verbale de la douleur chez 47 patients français et 34 syriens atteints de leucémie. L'intensité (EVA) et les dimensions sensorielle et affective (QDSA) de leur douleur étaient recueillies. Les descripteurs sensoriels et émotionnels choisis par les syriens, ont un contenu sémantique plus fort que ceux utilisés par les français. L'origine culturelle du patient, en modifiant la qualité sémantique de l'expression verbale de la douleur, doit être prise en considération dans les pratiques de soins.

Mots clés : évaluation de la douleur, cancer, culture, étude interculturelle. 
Selon Boureau [1], la douleur est composée de sensations complexes, à la fois physiques et émotionnelles. Ses syndromes sont définis comme "l'ensemble des facteurs émotionnels, cognitifs et comportementaux susceptibles de participer à l'entretien et à l'exacerbation d'une douleur chronique » [2]. Ainsi, le modèle « multidimensionnel » par ses composantes: sensori-discriminative, affective-émotionnelle, cognitive et comportementale aide à une meilleure compréhension de la douleur [3]. Au cours des entretiens d'évaluation de la douleur, nous avons demandé aux patients d'utiliser des descripteurs sensoriels et émotionnels pour décrire leur douleur, afin de mieux appréhender la prise en charge de la douleur lors du processus de soin. En effet, la recherche d'un syndrome douloureux doit être organisée, et l'évaluation de ce syndrome va permettre d'expliquer le rapport de causalité avec la pathologie, de prévoir certaines difficultés et de développer un projet thérapeutique.

La douleur est une expérience subjective, intime, influencée par de nombreuses variables dont la culture. Selon Cathébras [4], la culture peut déterminer l'attitude et le comportement face à la douleur, à travers les représentations, et les croyances attachées à celle-ci. La culture représente un système de savoirs qui permet à un individu, à un groupe ou une société de fonder des modèles explicatifs sur les causes, les symptômes, l'évolution et le traitement d'une maladie [5]. Le comportement-maladie a été défini comme "une expérience normative gouvernée par des règles culturelles : nous apprenons des manières "approuvées" d'être malade » [6].

Les personnes attribuent à leur douleur un sens et une valeur différente selon les orientations collectives propres au milieu dans lequel ils vivent [7]. Ainsi, les catégories de pensées et de perceptions, qui soulèvent la crainte ou l'indifférence, sont établies par le modèle culturel auquel l'individu appartient [8]. Dans ce contexte, la douleur n'est pas seulement un événement biologique brut mais elle représente aussi une signification relative à un individu. Ces significations sont induites par le rapport du patient à la douleur et le sens qu'il lui donne. Ce sens s'évalue à travers l'histoire de patient, sa culture, ses règles sociales ainsi que son contexte [9]. Ainsi, la relation entre douleur et culture soulève le problème de l'interaction complexe des facteurs biologiques, psychologiques, culturels et sociaux dans la perception et l'expression de la douleur [4]. Les différences entre les cultures sont quelquefois attachées aux valeurs attribuées à la douleur. Les résultats de l'étude quantitative de Bates, Edwards, \& Anderson [10] confirment que l'intensité de la douleur varie selon les attitudes et les croyances associées à différents groupes ethniques. La culture ne détermine alors ni la composante sensorielle ni la composante émotionnelle de la douleur de façon significative. Cependant, les références culturelles peuvent donner lieu à différents styles d'expression de plaintes et influencer la perception de la douleur [11]. Ainsi, la dimension culturelle influence les perceptions sensorielles de la douleur et donne différents scores d'intensité. Aucune différence n'est observée dans l'intensité de la douleur entre les différentes cultures, mais une distinction s'observe quant à sa tolérance. Il y a des interactions complexes entre la culture et la manière dont les individus perçoivent et expriment la douleur.

L'impression dominante est que les personnes d'origine culturelle différente vivent et expriment leurs douleurs de manières diverses. Ainsi, les Méditerranéens, dans l'ensemble plutôt affectifs, auraient des tendances à exagérer leur douleur. Par contre, les Britanniques auraient une sensibilité très élevée et une tendance à moins se plaindre [12]. Pour éviter les stéréotypes culturels, il est nécessaire de partir du postulat que les différences rencontrées au sein d'une culture sont parfois plus marquées que celles qui distinguent les cultures entre elles [13].

Toutes les sociétés confirment implicitement la légitimité de la douleur liée à des particularités sociales, culturelles ou physiques pénibles [14]. Dans cette recherche, nous prenons en compte l'importance culturelle de la douleur. Les relations entre douleur et culture posent la question de savoir comment l'expérience douloureuse peut être traduite et communiquée dans le cadre d'une relation thérapeutique. Notre travail vise à explorer la question de l'expression verbale de la douleur au regard de l'origine culturelle du patient. Les patients adultes atteints d'une même maladie ou du même syndrome algique, utilisent des descripteurs pour communiquer ce qu'ils ressentent sans que 
soient pris en considération leurs origines culturelles. L'objectif de notre étude est, en comparant les sociétés syrienne et française, d'étudier l'influence de la culture sur l'expression verbale de la douleur. Nous avons choisi ces deux cultures parce qu'il y a une grande différence concernant la représentation sociale qui se manifeste dans les productions langagières de la douleur. En fait, dans la culture syrienne, la prise en charge de la douleur n'est pas considérée comme prioritaire par l'Etat. Le soutien psychologique du patient est assuré par les proches [15]. En revanche, dans la culture française, la prise en charge de la douleur est un objectif de santé publique régi par la Loi depuis plusieurs décennies (article 1110-5 du code de santé publique).

Nous cherchons à savoir si l'expression verbale de la douleur est fonction de la culture d'origine des patients.

A partir de cette problématique, nous émettons l'hypothèse que les patients ressentent la douleur à des degrés différents et que la culture jouerait un rôle qui peut différencier le lexique des patients souffrants. En effet, il semble important dans une approche culturelle, d'une part, et clinique concernant la prise en charge, d'autre part, de saisir la manière dont les patients vivent les différentes expériences de la douleur, et les moyens expressifs qu'ils mettent en œuvre pour la verbaliser en prenant en compte l'influence de la culture locale. Nous formulons l'hypothèse que :-le vocabulaire dans ses composantes sensorielles et émotionnelles de la douleur serait discriminant : les syriens utiliseraient plus de métaphores sensorielles de la douleur et emploient plus de descripteurs affectifs que les français.

\section{Méthodologie}

L'intérêt de cette recherche est de tenter d'améliorer l'évaluation de l'intensité de la douleur pour une prise en charge plus efficace des patients.

Les entretiens d'évaluation de la douleur ont été effectués en France et en Syrie entre janvier 2010 et février 2011. Notre population se compose de 34 Syriens et 47 Français âgés de 45 à 65 ans, atteints d'une leucémie à un stade avancé métastatique sous traitement curatif. Sur les 34 sujets syriens, 15/34 sont des hommes, et sur les 47 français, 27/47 sont des hommes.

L'application des outils d'évaluation est individuelle et dure environ 15 minutes par patient. Tout d'abord, nous commençons par mesurer la douleur ressentie par le sujet en utilisant le questionnaire Douleur Saint-Antoine (QDSA). Ce questionnaire comprend 58 lexiques et 16 classes, les 9 premières représentent les aspects sensoriels de la douleur et les 7 dernières, les aspects affectifs. Dans chaque catégorie de descripteurs, le patient choisi les mots les plus proches de son ressenti et précise sa réponse en donnant au qualificatif qu'il choisit une note de 0 à $4(0=$ pas du tout, $4=$ extrêmement). Ensuite, nous procédons à l'enregistrement de la douleur actuelle du patient à l'aide de l'échelle visuelle analogique (EVA). Cette échelle permet de quantifier la douleur des patients à l'aide d'une échelle entre 0 (pas de douleur) et 10 (douleur extrême). Nous présentons au patient le côté de la réglette où il n'y a pas de chiffres. Le curseur de la réglette doit alors être positionné sur le zéro, uniquement visible par l'examinateur, et sera déplacé seulement par le patient. Ainsi, nous mesurons l'intensité de la douleur (forte, modérée et faible).

\section{Résultats}

-Echelle visuelle analogique (EVA) : Les scores des patients dans les deux cultures sont compris entre «modérés » (de 4 à 7$)$ et « forts » (de 8 à 10). Les moyennes des scores entre les femmes et les hommes français (respectivement 6,06/10 et 6,11/10) sont comparables alors que l'écart est plus important entre les femmes et les hommes syriens (respectivement 7,37/10 et 5,87/10). Par contre, aucune différence n'est observée dans l'intensité de la douleur entre les deux cultures françaises et syriennes. 
-Echelle du Questionnaire Douleur Saint-Antoine (QDSA): Selon Boureau [2], quatre types de scores peuvent être calculés pour la dimension sensorielle (de $\mathrm{A}$ à $\mathrm{I}$ ), affective (de $\mathrm{J}$ à $\mathrm{P}$ ) et pour les deux dimensions réunies (de $\mathrm{A}$ à $\mathrm{P}$ ) :

- le score correspondant au «nombre de classes choisies » (NCC) renvoie au nombre de mots sélectionnés dans chacune des classes composant le QDSA

- le «score valeur de rang» (SVR) est issu de la notation qui est induite par l'ordre d'apparition du terme choisi dans la liste (exemple: si dans la classe A, le sujet choisi le mot « élancement», la valeur de rang correspond à 3 puisque ce terme apparaît en troisième position). Cet ordre d'apparition du terme choisi reflète le degré d'intensité

- le «score note patient » (SNP) est calculé à partir de la note d'intensité, entre 0 (absent) et 4 (extrêmement fort), donnée par le patient sur le terme choisi le plus significatif de la classe (exemple: pour le terme «élancement» le patient peut attribuer un score d'intensité de 4, correspondant à extrêmement fort)

- le « score notes moyennes » (SNM) est la moyenne des deux précédents scores (score de rang et score note).

Dans notre étude, pour le score NCC, les descripteurs choisis (sensoriels, émotionnels et les deux ensembles) sont plus nombreux chez les syriens que ceux choisis par les français. Chez les femmes syriennes, la moyenne du score total est de 9,4/16, contre 6,1/16 chez les françaises (différence significative selon le résultat de l'ANOVA $F=30,95, p<0,05)$. Chez les hommes syriens, la moyenne du score total est de 9,13/16 contre 6,55/16 chez les français (différence significative $\mathrm{F}=16,06$, $\mathrm{p}<0,05)$ Dans ce contexte, nous pouvons affirmer que les sujets syriens décrivent leur mal concernant la composante sensorielle et émotionnelle par des termes plus variés que les patients français des deux sexes.

Pour le score SVR, les patients syriens obtiennent un résultat sémantique plus élevé que celui des français (aspect sensoriel et/ou émotionnel). Ainsi, cette moyenne est significativement plus élevée chez les femmes syriennes $(21,94 / 58)$ que chez les femmes françaises $(11,7 / 58)(\mathrm{F}=40,65, \mathrm{p}<0,05)$. De plus, les hommes syriens sélectionnent des lexiques à connotation plus intense, tant sensorielle qu'émotionnelle, en comparaison avec les hommes français. Le facteur culturel joue donc un rôle primordial sur la qualité sémantique des mots choisis par les patients syriens et français pour exprimer leur douleur.

Quant au score SNP, les patients syriens montrent globalement pour chaque terme une intensité de la douleur plus élevée comparativement aux patients cancéreux français. Ces différences sont confirmées par la moyenne totale des deux dimensions, sensorielles et émotionnelles, qui est de 22,05/64 chez les femmes syriennes tandis qu'elle est de 14,1/64 chez les femmes françaises. Les différences sont significatives sur l'aspect sensoriel, émotionnel et les deux variables confondues chez les patients syriens.

Pour le score SNM, les patients syriens montrent des scores très élevés comparativement aux patients français tant au niveau sensoriel qu'émotionnel que pour les deux regroupés. Le score moyen de la description sensorielle et émotionnelle est de 22/61 chez les Syriennes contre 12,9/61 chez les Françaises $(\mathrm{F}=41,01, \mathrm{p}<0,05)$. Pour l'aspect sensoriel du SNM, la moyenne est pratiquement le double chez les femmes syriennes $(11,97 / 35,5)$ que chez les françaises $(6,2 / 35,5)$. Pour l'aspect affectif, le score moyen des femmes syriennes $(10,02 / 25,5)$ est nettement plus élevé que celui des françaises $(6,7 / 25,5)$. De plus, des différences similaires ont été constatées entre les hommes syriens et français en ce qui concerne le SNM sur l'aspect sensoriel et affectif ainsi que sur les deux aspects ensembles. Notre hypothèse de l'influence du facteur «culture » sur la perception et l'expression verbale de la douleur est confirmée. Le vocabulaire utilisé par les patients français et syriens pour exprimer leur douleur au niveau sensoriel et au niveau émotionnel est un élément discriminant à prendre en compte dans les pratiques de soin. 


\section{Discussion}

Dans notre étude, l'intensité de la douleur varie entre des valeurs « modérées » et « fortes » chez la plupart des patients des deux cultures selon l'échelle de la douleur EVA. Globalement, les évaluations « douleur modérée » sont fréquentes dans ces populations. L'enquête d'European Pain In Cancer (EPIC), menée dans 12 pays européens, montre que la douleur cancéreuse est une évidence en France où la majorité des patients concernés (76\%) souffre de douleurs attribuées à leur cancer. L'échelle EVA est actuellement l'outil le plus utilisé par le corps médical pour appréhender et prendre en charge la douleur des patients de part sa facilité d'emploi. Dans notre étude, nous n'avons pas mis en évidence de différence significative de moyenne de score d'EVA entre le groupe de patients syriens et le groupe français : l'intensité de la douleur ressentie par les patients français et par les patients syriens serait donc comparable au sein de notre étude si l'évaluation de la douleur s'arrêtait à l'étude de l'échelle EVA. L'échelle EVA ne permet pas de prendre en considération le facteur culturel dans la perception de la douleur.

La douleur constitue une expérience subjective. Sa dimension sensorielle et émotionnelle est universellement acceptée. L'émotion n'est plus considérée comme une simple conséquence de la douleur mais au contraire, comme un élément fondamental de la douleur ressentie. Selon notre étude, avec l'échelle QDSA, les patients douloureux syriens et français (hommes et femmes) décrivent leur douleur ressentie en utilisant un lexique assez varié et en produisant des expressions aussi bien conventionnelles qu'originales. Selon Rollman [16], les réponses à la douleur suggèrent de possibles différences ethnoculturelles pour les deux facteurs affectifs et sensoriels. A l'inverse, pour Lipton \& Marbach [17], il est impossible d'avoir des formes d'expressions indépendantes entre différents groupes ethniques. Selon Greenwald [18], les liens significatifs sont absents entre l'identité ethnique et la dimension sensorielle de la douleur, mais ils sont identifiés entre les groupes ethniques et la dimension affective.

De même, l'attitude des patients français et des patients syriens envers leur douleur ressentie est caractérisée par des différences interculturelles concernant la description de la douleur. Dans notre étude, nous constatons que l'expression verbale de la douleur des patients syriens est plus importante que celle des patients français. Par conséquent, les patients syriens sélectionnent plus de descripteurs sensoriels et affectifs (moyenne Syrie $=9,2$ vs moyenne France $=6,4$ ). Une étude de Zola [19] a montré que les patients italiens se focalisent sur leurs problèmes et ont une tendance à exprimer davantage leurs plaintes tandis que les patients irlandais se plaignent peu. La recherche de Koopmana, Eisenthalb, \& Stoecklea [8] confirment que, les malades italiens évoquent exagérément des symptômes de maladie alors que leurs homologues américains sont plus réservés pour le même type de pathologie. Dans notre recherche, les descripteurs sensoriels et émotionnels, qui sont utilisés par les patients syriens, ont un contenu sémantique plus fort que ceux sélectionnés par les français (ex. l'expression « coupure » est largement utilisée chez les patients français, tandis que la réponse « coup de poignard» est assez répandue chez les patients syriens). Enfin, l'intensité de la douleur concernant chaque qualificatif est plus forte chez les patients syriens (moyenne Syrie $=21,17$ vs. moyenne France $=14,89$ ).

Nous proposons plusieurs explications à ces différences d'expression verbale de la douleur : -La langue arabe syrienne est plus variée dans le domaine de la douleur que la langue française -Le mode de communication syrien, basé sur la répétition, l'insistance et l'exagération, induit artificiellement un plus grand volume de production, (ex. les métaphores, une longue liste d'adjectifs est utilisée pour modifier un simple nom et en souligner la signification) [20] -Le poids de l'histoire syrienne avec ses drames. L'utilisation plus courante en France qu'en Syrie des antalgiques ne semble pas en cause puisque dans notre étude, aucune distinction d'intensité de douleur n'a été mise en évidence avec l'échelle EVA entre les deux cultures. 
Notre conception de la douleur part d'une vision multidimensionnelle. Nous nous sommes intéressés à l'influence de la culture sur l'expression verbale de la douleur. Notre objectif était de montrer l'importance de la culture dans l'expression verbale sensorielle et émotionnelle de la douleur. Dans les deux populations étudiées, le lexique descriptif de la douleur s'avère riche. Toutefois, pour tous les indices douloureux étudiés par l'échelle QDSA, l'expression de la douleur est plus importante chez le sujet syrien que chez le sujet français. Généralement, les niveaux de la perception et de l'expression de la douleur sont influencés par l'origine culturelle du patient et par son environnement social et familial. Il semble nécessaire afin d'arriver à une prise en charge efficace du contrôle de la douleur pour tous les patients, de prendre en considération leur origine culturelle.

Les outils que nous avons utilisés constituent une étape majeure dans la prise en compte des patients. En effet, toutes les expressions de la douleur ne peuvent pas être appréhendées uniquement par l'Echelle Visuelle Analogique EVA, et il nous a semblé intéressant de réaliser une exploration plus poussée en employant des échelles qui portent sur l'évaluation subjective de la douleur. L'évaluation de l'expression verbale de la douleur dans sa dimension affective et sensorielle par le QDSA nous a permis de mieux appréhender la douleur ressentie par les patients syriens. L'utilisation de ces différents outils entraînent « de facto » une reconnaissance de l'importance de la personne malade et de ses propos pouvant entraîner un changement favorable dans la relation médecin /malade et amener l'équipe de soin à pondérer dans un sens ou un autre les résultats des différentes échelles selon l'origine culturelles des patients. Comment à la faveur du phénomène douloureux amplifier cette réflexion de la place du malade dans le parcours de soin ? Cette douleur qui s'inscrit dans un contexte de maladie du cancer ne relève pas du même registre qu'une douleur liée à des problèmes de douleurs chroniques, tout simplement parce que le cancer résonne dans la plupart des cas comme une sentence de mort. La question de l'influence de la culture est déterminante dans toutes les manifestations douloureuses qui sont un rappel pour les malades de cette maladie qui peut peut être les emporter. C'est cette perception subjective, effectuée par le patient, qui est au centre de nos préoccupations. Nous espérons que notre travail de recherche permettra une ouverture sur la prise en charge de la douleur et contribuera à la mise en place de pratiques psychothérapeutiques pour les patients en souffrance. 


\section{Références bibliographiques}

1. Boureau, F. (1988). Pratique du traitement de la douleur. Paris: Doin.

2. Boureau, F., Luu, M., \& Doubrère, J. F. (1992). Comparative study of the validity of four French McGill Pain Questionnaire (MPQ) versions. Pain, 50 (1), pp. 59-65.

3. Serra, E., \& Verfaillie, F. (2007). Douleur et Souffrance: de quelques aspects médicaux. Ethique et Santé, 4, pp. 131-135.

4. Cathébras, P. (2000). Douleur, somatisation, et culture : Peut-on aller au-delà des stéréotypes? Doul et Analg, 3, pp. 159-162.

5. Fischer, G. N., \& Tarquinio, C. (2006). Les concepts fondamentaux de la psychologie de la santé. Paris: Dunod.

6. Kleinman, A., Eisenberg, L., \& Good, B. (1978). Culture, Illness, and Care: Clinical Lessons from Anthropologic and Cross-Cultural Research. Annals of Internal Medicine, 88 (1), pp. 251258.

7. Cedraschi, C. (2003). Aspects anthropologiques et socioculturels des représentations de la douleur chronique. Dans J. Wrobel, Aspect psychologique de la douleur chronique (pp. 106-118). Paris.

8. Cathébras, P. (2009). Diversité culturelle et expression de la douleur en rhumatologie. Revue $d u$ rhumatisme, 76 (6), pp. 517-521.

9. Bates, M. S., Edwards, W. T., \& Anderson, K. O. (1993). Ethnocultural influences on variation in chronic pain perception. Pain, 52 (1), pp. 101-112.

10. Morris, D. B. (2001). L'ethnicité et la douleur. Pain Clinical Updates, IX (4), pp. 1-4.

11. Wolff, B. B. (1985). Ethnocultural Factors Influencing Pain and Illness Behavior. Clinical Journal of Pain, 1 (1), pp. 23-30.

12. Weisenberg, M., \& Caspi, Z. (1989). Cultural and Educational Influences on Pain of Childbirth. Journal of Pain and Symptom Management, 4 (1), pp. 13-19.

13. Choinière, M., \& Melzack, R. (1987). Acute and chronic pain in hemophilia. Pain, 31 (3), pp. 317-31.

14. Moore, R., Brodsgaard, I., Mao, T. K., Miller, M., \& Dworkin, S. F. (1998). Acute Pain and Use of Local Anesthesia: Tooth Drilling and Childbirth Labor Pain Beliefs Among AngloAmericans, Chinese, and Scandinavians. Anesth Prog , 45, pp. 29-37.

15. Atallah, F., \& Guillermou, Y. (2004). L'homme et sa douleur : dimension anthropologique et sociale. Annales françaises d'anesthésie et de réanimation, 23 (7), pp. 722-729.

16. Le Breton, D. (1995). Anthropologie de la douleur. Paris: Métailié.

17. Rollman, G. B. (2005). The need for ecological validity in studies of pain and ethnicity. Pain, $113(1)$, pp. 3-4.

18. Lipton, J., \& Marbach, J. (1984). Ethnicity and the pain experience. Soc Sci Med, 19 (12), pp. 1279-1298.

19. Zola, I. K. (1966). Culture and Symptoms--An Analysis of Patient's Presenting Complaints. American Sociological Review, 31 (5), pp. 615-630.

20. Feghali, E. (1997). Arab Cultural Communication Patterns. Elsevier Science, 21 (3), pp. 345-378. 